\title{
Regulatory control circuits for stabilizing long-term anabolic product formation in yeast
}

D'ambrosio, Vasil; Dore, Eleonora; Di Blasi, Roberto; van den Broek, Marcel; Sudarsan, Suresh; ter Horst, Jolanda; Ambri, Francesca; Sommer, Morten Otto Alexander; Rugbjerg, Peter; Keasling, Jay D. Total number of authors:

12

Published in:

Metabolic Engineering

Link to article, DOI:

10.1016/j.ymben.2020.07.006

Publication date:

2020

Document Version

Publisher's PDF, also known as Version of record

Link back to DTU Orbit

Citation (APA):

D'ambrosio, V., Dore, E., Di Blasi, R., van den Broek, M., Sudarsan, S., ter Horst, J., Ambri, F., Sommer, M. O. A., Rugbjerg, P., Keasling, J. D., Mans, R., \& Jensen, M. K. (2020). Regulatory control circuits for stabilizing long-term anabolic product formation in yeast. Metabolic Engineering, 61, 369-380.

https://doi.org/10.1016/j.ymben.2020.07.006

\section{General rights}

Copyright and moral rights for the publications made accessible in the public portal are retained by the authors and/or other copyright owners and it is a condition of accessing publications that users recognise and abide by the legal requirements associated with these rights.

- Users may download and print one copy of any publication from the public portal for the purpose of private study or research.

- You may not further distribute the material or use it for any profit-making activity or commercial gain

- You may freely distribute the URL identifying the publication in the public portal 


\title{
Regulatory control circuits for stabilizing long-term anabolic product formation in yeast
}

\author{
Vasil D’Ambrosio $^{\mathrm{a}, 1}$, Eleonora Dore ${ }^{\mathrm{a}, 1}$, Roberto Di Blasi ${ }^{\mathrm{a}}$, Marcel van den Broek ${ }^{\mathrm{b}}$, \\ Suresh Sudarsan ${ }^{a}$, Jolanda ter Horst ${ }^{a}$, Francesca Ambri ${ }^{a}$, Morten O.A. Sommer ${ }^{a}$, \\ Peter Rugbjerg $^{\mathrm{a}}$, Jay D. Keasling ${ }^{\mathrm{a}, \mathrm{c}, \mathrm{d}, \mathrm{e}, \mathrm{f}}$, Robert Mans ${ }^{\mathrm{b}, * *}$, Michael K. Jensen ${ }^{\mathrm{a}, *}$

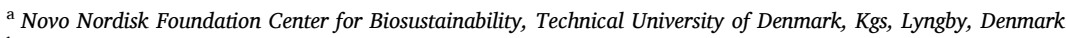 \\ ${ }^{\mathrm{b}}$ Department of Biotechnology, Delft University of Technology, Delft, the Netherlands \\ c Joint BioEnergy Institute, Emeryville, CA, USA \\ ${ }^{\mathrm{d}}$ Biological Systems and Engineering Division, Lawrence Berkeley National Laboratory, Berkeley, CA, USA \\ ${ }^{\mathrm{e}}$ Department of Chemical and Biomolecular Engineering, Department of Bioengineering, University of California, Berkeley, CA, USA \\ ${ }^{\mathrm{f}}$ Center for Synthetic Biochemistry, Institute for Synthetic Biology, Shenzhen Institutes of Advanced Technologies, Shenzhen, China
}

\section{A R T I C L E I N F O}

\section{Keywords:}

Stability

Yeast

Production

Biosensor

Essential genes

Control circuits

\begin{abstract}
A B S T R A C T
Engineering living cells for production of chemicals, enzymes and therapeutics can burden cells due to use of limited native co-factor availability and/or expression burdens, totalling a fitness deficit compared to parental cells encoded through long evolutionary trajectories to maximise fitness. Ultimately, this discrepancy puts a selective pressure against fitness-burdened engineered cells under prolonged bioprocesses, and potentially leads to complete eradication of high-performing engineered cells at the population level. Here we present the mutation landscapes of fitness-burdened yeast cells engineered for vanillin- $\beta$-glucoside production. Next, we design synthetic control circuits based on transcriptome analysis and biosensors responsive to vanillin- $\beta$-glucoside pathway intermediates in order to stabilize vanillin- $\beta$-glucoside production over $\sim 55$ generations in sequential passage experiments. Furthermore, using biosensors with two different modes of action we identify control circuits linking vanillin- $\beta$-glucoside pathway flux to various essential cellular functions, and demonstrate control circuits robustness and almost 2-fold higher vanillin- $\beta$-glucoside production, including 5-fold increase in total vanillin- $\beta$-glucoside pathway metabolite accumulation, in a fed-batch fermentation compared to vanillin$\beta$-glucoside producing cells without control circuits.
\end{abstract}

\section{Introduction}

To support sustainable and environmentally friendly productionprocesses, research focuses on the production of bio-based alternatives to petroleum-based production processes, made by engineered cell factories (Kruyer and Peralta-Yahya, 2017). However, in order to design commercially attractive bioprocesses for converting cheap renewable substrates into value-added chemicals, innovative bioprocesses need to be developed. For this purpose, online monitoring and sampling is essential in modern fermentation processes, in order to control and optimize bioreactor conditions for biobased production (Gomes et al., 2018). Usual parameters analysed online include $\mathrm{pH}$, exhaust $\mathrm{CO}_{2}$, temperature, aeration, agitation, and dissolved oxygen. However, most often, any of these parameters are mere proxies for evaluating the actual biocatalysis, i.e. the microbial production of a chemical or protein of interest, which is often analysed offline during or following completion of the actual fermentation (Gomes et al., 2018).

The lack of monitoring and control of culture performance becomes critical as bioreactor volume and bioprocess duration increase. This is because biobased production over prolonged cultivation regimes is especially challenged by culture take-over by mutated, low-performing cells with increased specific growth rates (Rugbjerg and Sommer, 2019; Wang and Dunlop, 2019; Xiao et al., 2016). Indeed, nongenetic and genetic heterogeneity of microbial populations is largely

\footnotetext{
* Corresponding author.

$* *$ Corresponding author.

E-mail addresses: R.Mans@tudelft.nl (R. Mans), mije@biosustain.dtu.dk (M.K. Jensen).

1 These authors contributed equally to this study.
} 
acknowledged as evident from large differences in growth rate, resistance to stress, and regulatory circuit output of isogenic populations (Carlquist et al., 2012; Müller et al., 2010; Rugbjerg et al., 2018b). Even more so, in industrial high-cell density fed-batch bioprocesses, subpopulations exist that are many-fold different in these parameters from the population average (Wang and Dunlop, 2019; Xiao et al., 2016). These random variations arise because not all cells are exactly of the same size, some cells may have been mutated during prolonged seed trains and growth in large-scale cultivations, and nor do all cells have the same number of key components, incl., RNA polymerase, ribosomes, and other key factors governing the life of a cell (Elowitz et al., 2002; Müller et al., 2010; Rugbjerg et al., 2018b). In fact, such random variations and evolutionary drifting can give rise to low-performing variants accounting for up to $80-90 \%$ of the total cell population, and produce less than half of the desired product (Xiao et al., 2016). Thus, there is a strong motivation for developing new technologies that can monitor biocatalysts and optimize bioprocesses by coupling detectable phenotypes to product formation in an efficient manner.

Small-molecule biosensors offer sensitive and real-time monitoring of product formation in microbial cell factories (David et al., 2016; Tao et al., 2017; Zhang et al., 2016), and are furthermore emerging as a promising technology for safeguarding high-performing productive cell factories from evolutionary drifting into low-performing ensembles during prolonged cultivations, as those often applied in industry (Rugbjerg et al., 2018b). Specifically, biosensors based on allosterically regulated bacterial transcription factors undergo conformational changes upon binding of specific intracellular ligands, and can directly couple single-cell ligand accumulation to a change in reporter gene expression (e.g. fluorescence or antibiotic resistance)(Fernandez-López et al., 2015). Biosensors detecting small-molecule accumulation can be employed for facile evaluation of subpopulation heterogeneity in diverse feedstock and bioreactor environments, and can support the prototyping of optimal bioreactor conditions in relation to production of any candidate chemical for which a biosensor is available (Flachbart et al., 2019; Snoek et al., 2018; Xiao et al., 2016). Also, as the biosensors can couple product accumulation with gene expression output, they not only allow for monitoring of product accumulation at the single-cell level (diagnosis), but have also been used to couple product accumulation to growth of bacterial cell factories, and thereby enable selective growth advantage of high-performing subpopulation variants and/or shunting of competing metabolic pathway reactions (therapy)(Rugbjerg et al., 2018b; Xiao et al., 2016).

In this study, we evaluate the coupling of fitness-burdening productformation to biosensor-controlled expression of essential genes covering four different metabolic functions of baker's yeast Saccharomyces cerevisiae engineered for production of industrially relevant vanillin$\beta$-glucoside. From this we demonstrate that yeast cells engineered with control circuits based on biosensors controlling essential gene expression are recalcitrant to evolutionary drifting over 50 generations. Ultimately, the best-performing control circuit is benchmarked with the parental vanillin- $\beta$-glucoside producing strain in a fed-batch fermentation, in which strains with control circuits accumulate 5 -fold higher total pathway intermediates, including approximately 2-fold higher final vanillin- $\beta$-glucoside levels, compared to the parental strain.

\section{Results}

\subsection{Selection and physiological characterization of production strain}

To investigate the ability of genetically-encoded biosensors to stabilize production of ATP-requiring molecules in yeast, first a proof-ofconcept production testbed was selected. Here we chose a $S$. cerevisiae cell factory engineered for the production of vanillin- $\beta$-glucoside (VG) (Strucko et al., 2015). Importantly, vanillin is the main molecule responsible for the vanilla flavour, and one of the most common nutraceutical compounds, used in food, pharmaceutical cosmetic, and other industries (Luziatelli et al., 2019) yet yeast strains carrying the vanillin- $\beta$-glucoside biosynthetic pathway have been shown to exhibit a reduced growth rate compared to non-producing $S$. cerevisiae (Supplementary Fig. S1)(Brochado et al., 2010; Hansen et al., 2009; Strucko et al., 2017, 2015). As for biosensors, we sought to employ two recently developed biosensors for VG pathway intermediates, namely PcaQ from Sinorhizobium meliloti for protocatechuic acid (PAC) detection, and VanR from Caulobacter crescentus for vanillic acid (VAC) detection (Ambri et al., 2020).

The VG producing strain used in this study carries single-copy genomic integration of five heterologous genes required for the de novo biosynthesis of vanillin- $\beta$-glucoside in $S$. cerevisiae: 3-dehydroshikimate dehydratase (3DSD) from Podospora anserina, O-methyltransferase (OMT) from Homo sapiens, aromatic carboxylic acid reductase (ACAR) from Nocardia iowensis, phosphopantetheine transferase (EntD) from Escherichia coli and UDP-glycosyltransferase (UGT) from Arabidopsis thaliana (Brochado et al., 2010; Hansen et al., 2009). Furthermore, the alcohol dehydrogenase $A D H 6$ and the $\beta$-glucosidase BGL1 genes were removed to further optimize the pathway (Hansen et al., 2009). The first step converts the native shikimate pathway intermediate 3-dihydroshikimate (3-DHS) to PAC, which is then converted to vanillin (VAN) through the heterologous intermediates VAC and protocatechuic aldehyde (PAL). Vanillin is finally converted to vanillin- $\beta$-glucoside to alleviate vanillin toxicity and improve product secretion (Brochado et al., 2010; Hansen et al., 2009; Strucko et al., 2015) (Fig. 1A and B).

In order to identify potential evolutionary pressure points for genetic instability of strains expressing the fitness-burdening VG pathway, we constructed single knockout variants of each of the five genes encoding the VG pathway enzymes, and tested the specific growth rates on synthetic medium permissive for VG formation of all resulting mutant strains compared to the parental VG producing strain with all five genes left intact (Fig. 1C, Supplementary Fig. S2). From this analysis we found that deletion of the 3DSD gene (VG-3DSD $\Delta$ ), encoding the enzyme catalysing the conversion from 3DHS to PAC, resulted in increased maximum specific growth rate and shortened lag phase compared to the full VG pathway strain, whereas deletion of any of the other genes in the pathway resulted in mutants with reduced growth rates compared to the parental VG pathway strain (Fig. 1C and D). Furthermore, even though the growth rate noticeable differs only between the VG-3DSD $\Delta$ and VG pathway strain, we observed that when ACAR, OMT or EntD genes are deleted, the lag phase is significantly longer, suggesting that the introduction of the pathway redirects the flux of metabolites from the biosynthesis of aromatic amino acids to the pathway causing a delay in the exponential growth phase.

Combined, these results indicate that depletion of shikimate intermediates and/or build-up of VG pathway intermediate PAC impose a fitness burden to the cells, whereas the single loss-of-function of any other pathway gene results in growth disadvantage, corroborating earlier reports on vanillin toxicity and the response of $S$. cerevisiae to weak acids and lignin derivatives (Gu et al., 2019; Guo and Olsson, 2014; Hansen et al., 2009).

\subsection{Assessment of cell factory stability}

Having evaluated the burden of the VG pathway on host fitness based on systematic pathway truncations, we next sought to investigate VG pathway metabolite profiles and pathway stability of the VG production strain. For this purpose, a serial passaging experiment consisting of seven sequential transfers ( $\sim 55$ generations) was performed. Here cultures were initially grown in YPD medium and then transferred in synthetic medium (SM) (Verduyn et al., 1992) to acclimate them to the experimental conditions (Fig. 2A). From this experiment we initially observed that following first transfer, in which cultures were diluted 1:100 and grown for $48 \mathrm{~h}$ in fresh medium, the extracellular metabolite content was biased towards PAC, being the first intermediate of the VG pathway, accumulating to approx. $2 \mathrm{mM}$, while end-product VG 
A

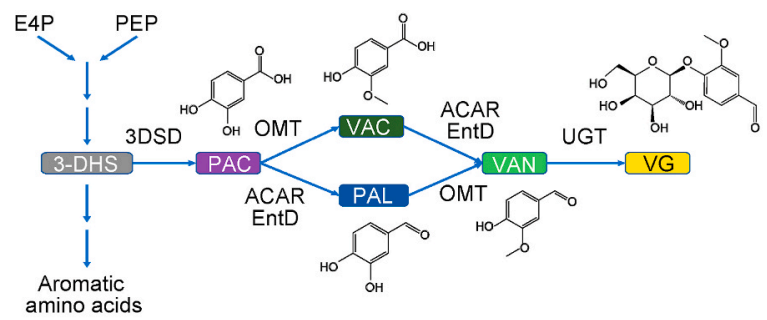

C

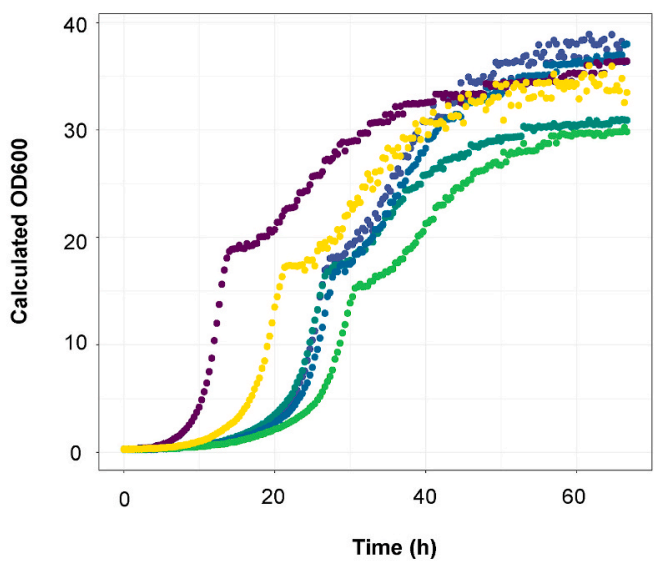

B

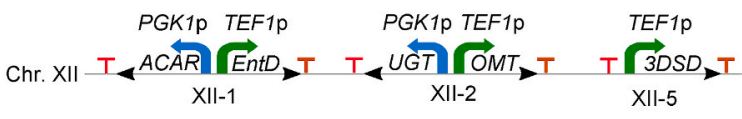

D

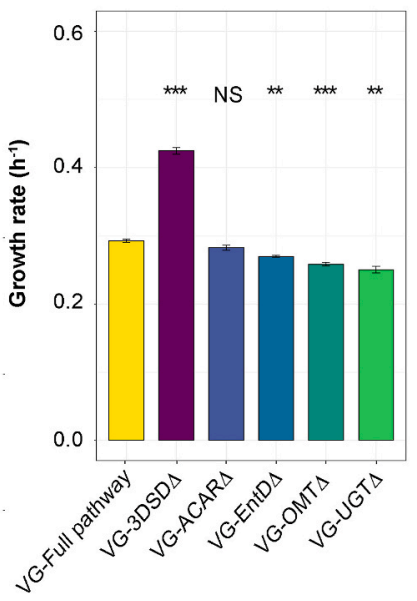

Fig. 1. Identification of fitness-burdening reactions in the vanillin- $\beta$-glucoside biosynthetic pathway.

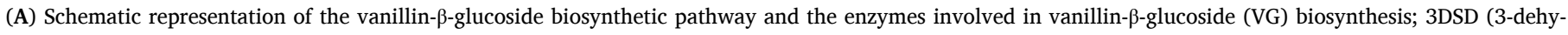

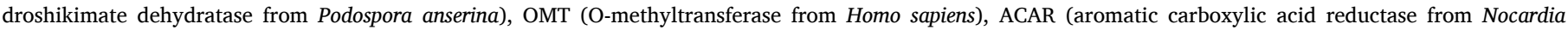

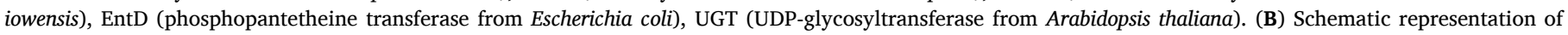

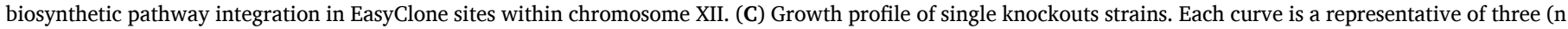

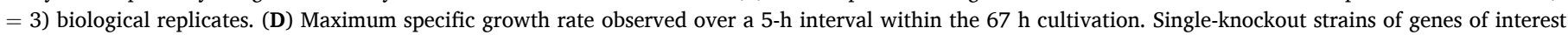

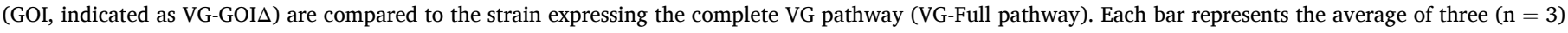

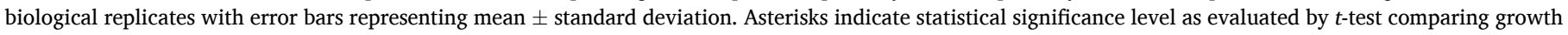

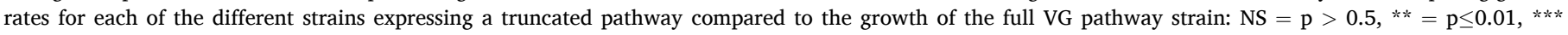
$=\mathrm{p} \leq 0.001$.

accumulated to $0.78 \mathrm{mM}$ (Fig. 2B). This observation agrees with the original VG pathway study performed on this strain (Strucko et al., 2015). For each of the following transfers, cultures were further diluted 1:100 every $48 \mathrm{~h}$ into fresh medium, thus initiating the next batch phase (Fig. 2A). At the end of each transfer, samples were collected for quantification of all pathway intermediates and VG end-product formation in order to assess the total flux through the VG pathway in each batch phase of the passage regime. Following the second transfer, a $46 \%$ drop in PAC productivity was observed compared to transfer 1 . This result is in line with the sequencing of the 3DSD gene from seven single colonies from this transfer where 4 out of 7 sequences were mutated (Supplementary Table S4). Ultimately resulting in the inability to detect any extracellular PAC by the end of the fourth transfer. Similarly, VAC decreased by $60 \%$ and $97 \%$ by the end of the second and fourth transfer, respectively (Fig. 2C). On the other hand, the decrease in VAN and VG concentrations was delayed compared to the other pathway intermediates. In the second transfer the extracellular concentration of VAN and VG only decreased by $24 \%$ and $6 \%$, respectively. However, by the end of the fourth transfer VAN and VG concentrations decreased by 81.5 and $91.4 \%$, respectively (Fig. 2C), whereas by the end of the experiment VG had decreased by $98.5 \%$.

Additionally, at the end of each sequential cultivation, part of the cultivation was stored and then proceeded we to evaluate the difference in growth between each transfer. Interestingly, we observed that while the productivity decreased there was a marked increase in growth rate, suggesting that the mutated strains were overtaking the cultivation

\section{(Supplementary Fig. S3).}

Based on the accumulated evidence, 3DSD activity and/or depletion of shikimate intermediates appear to be causing the main fitness burden when expressing the VG pathway in yeast (Fig. $1 \mathrm{C}$ and D and 2). For this reason, we sequenced the genomically integrated 3DSD gene of 22 isolated single colonies from the fourth transfer of six parallel sequential batch cultures. From the sequencing analysis, we observed 7 premature stop codons, 5 SNPs, and 8 recombination events between the $3 D S D$ gene and either the gene encoding EntD or OMT, all controlled by the same TEF1 promoter design and CYC1 terminator (Fig. 1B). The remaining 2 colonies sequenced did not have any mutations in the $3 D S D$ gene (Table 1).

Taken together, these results suggest that the fitness-burdened parental VG producing strain is genetically unstable, and that the burden exerted by the pathway is predominantly alleviated via mutations in the first enzymatic step of the pathway encoded by 3DSD, converting 3DHS into PAC. Moreover, the single knockout experiment shows that loss of any subsequent VG pathway gene in a strain with an intact $3 D S D$ gene negatively impacts the growth rate of the resulting truncated pathway designs (Fig. 1B).

\subsection{Biosensor candidates and characterization}

As already mentioned, control of population heterogeneity and stabilization of heterologous end-product formation has previously been established in bacteria by the use of small-molecule biosensors 
A

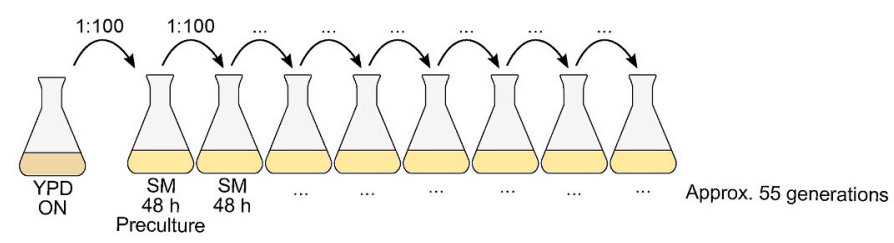

B

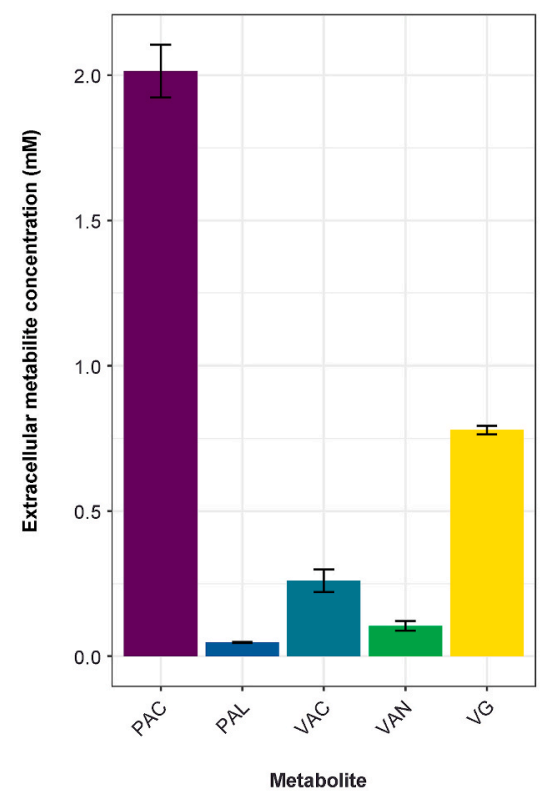

C

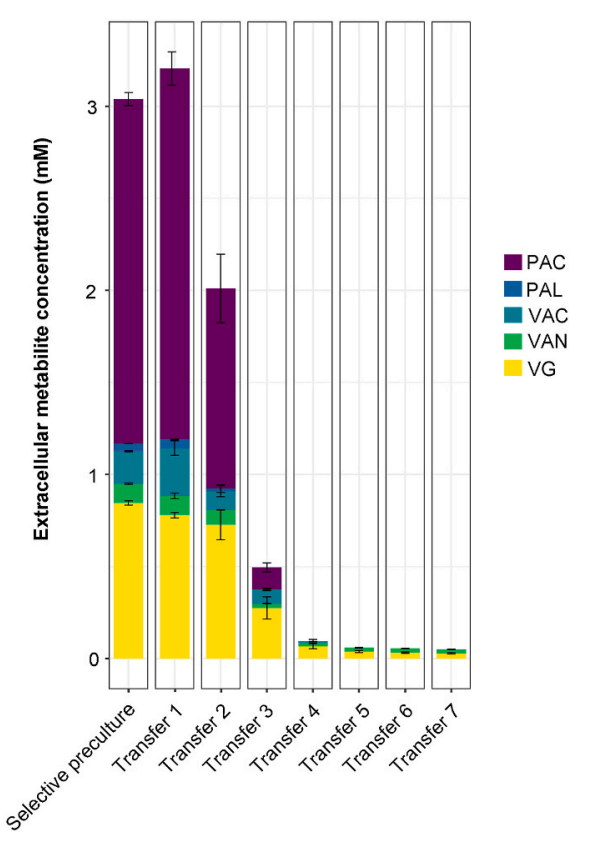

Fig. 2. Assessing vanillin- $\beta$-glucoside biosynthetic pathway stability during prolonged cultivation using metabolite profiling. (A) Schematic layout of the sequential transfer regime of batch cultures. The strains were initially cultivated in YPD overnight. Next, the cultures were diluted in synthetic medium to allow the strains to acclimate for continued growth in synthetic medium. (B) HPLC analysis of the extracellular metabolite concentrations for the VG strain following the first sequential passage. (C) Extracellular metabolite concentrations for the VG strain after $48 \mathrm{~h}$ cultivations over seven sequential transfers. The bars represent the average of two $(n=2)$ biological replicates. Error bars represent mean \pm standard deviation from two $(\mathrm{n}=2)$ biological replicates.

Table 1

List of identified mutations in the 3DSD gene. List of the identified mutations in the 3DSD gene at the end of the fourth sequential passage from six parallel cultivations. Each entry represents the result from one of the 22 sequenced colonies.

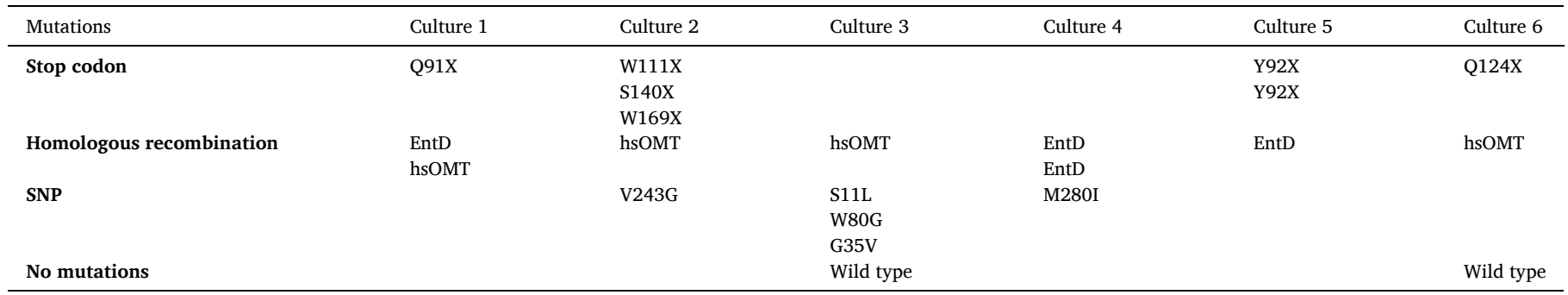

(Rugbjerg et al., 2018b; Xiao et al., 2016). In this study we wished to extend from this concept and assess the potential for control of production from heterologous pathways in eukaryotes making use of control circuits founded on prokaryotic small-molecule biosensors conditionally controlling expression of native genes essential to yeast. Moreover, we aimed to explore the hitherto unknown potential of engineering control circuits for stabilizing pathway intermediates instead of end product formation, thereby aiming to demonstrate the use of many more small-molecule biosensors for control circuit applications than possible if only considering biosensor-assisted control of end product formation.

For choice of biosensors we selected VanR from Caulobacter crescentus and PcaQ from Sinorhizobium meliloti, which we recently designed and applied as biosensors for VAC and PAC, respectively (Ambri et al., 2020; D'Ambrosio et al., 2020). Importantly, engineering control circuits by the use of either VanR or PcaQ, would enable testing of circuit performance founded on two different modes-of-action, potentially impacting the stability of the control circuits. Mechanistically, VanR is a transcriptional repressor which, in the absence of VAC, prevents transcription by binding to VanR operator sites (VanO) in promoters and thus confers sterical hindrance of RNA polymerase activity (Gitzinger et al., 2012). In the presence of VAC, VanR undergoes a conformational change, decreasing its affinity to VanO, upon which transcription can start (Jain, 2015). For the PAC biosensor, the transcriptional activator PcaQ, a LysR-type transcriptional regulator, constitutively binds PcaO operator sites in gene promoters and induces transcription of the output gene in the presence of PAC (Ambri et al., 2020; Fernandez-López et al., 2015). Regarding the design of the genetically-encoded biosensors, the VAC biosensor is composed of a bi-directional system where VanR is expressed under the control of the constitutive PGK1 promoter while the output gene is controlled by a synthetic VanO-containing TEF1 promoter (Ambri et al., 2020). The strong promoter controlling the expression of 
GFP allows for a high maximum ON state, while simultaneously, a strong expression of the allosterically regulated transcription factor allows for a strong repression and therefore a low OFF state. This design supports an operational range spanning $>2$ orders of magnitude of VAC concentrations, including the range of metabolite concentration observed in the parental VG strain (Fig. 2, and Supplementary Fig. S4). For the PAC biosensor, PcaQ is expressed from the strong constitutive TDH3 promoter, while the output gene is controlled by a truncated PcaO-containing CYC1 promoter (209 bp). This design allow for a very low expression, and therefore leakiness, in the absence of PAC, and a high dynamic output range when PAC is produced intracellularly. (Ambri et al., 2020).

To characterize VanR and PcaQ biosensors for sensing VAC and PAC concentrations, respectively, we first introduced VanR or PcaQ together with either GFP-expressing VanO- or PcaO-containing reporter promoters in both the VG production strain as well as in the non-producing VG-3DSD $\Delta$ strain, and measured fluorescence outputs. Here, the introduction of VanR and PcaQ in the VG strain resulted in increases in GFP read-outs of 2.7- and 3.5-fold, respectively, compared to their expression in the VG-3DSD $\Delta$ strain (Fig. 3), confirming that the biosensors are able to discriminate between VG-producing and non-producing strains.

\subsection{Evaluation of control circuit designs}

Spontaneous mutants with disruptive mutations in the $3 D S D$ gene, and therefore without the ability to produce VG and its biosynthetic intermediates, have a growth advantage compared to the strains harbouring the full VG pathway (Fig. 1C and D). Eventually, such differences in growth rate and lag phase will allow for complete population take-over of non-producer cells during prolonged cultivations. To extend the productive lifespan of a parental VG strain, we next sought to make use of the validated VAC and PAC biosensors for engineering control circuits in which mutants losing productivity will be subject to reduced fitness or even complete growth retardation. To do so, we decided to couple the expression of essential genes to the presence of VG pathway metabolites PAC and VAC, similar to previously reported studies in E. coli (Rugbjerg et al., 2018b; Xiao et al., 2016). Ideally, for such control circuits, only strains carrying a functional product pathway would be able to conditionally induce transcription of essential genes to a level sufficient for growth.

To select essential genes for control circuit designs, we performed a systematic analysis of candidate essential genes in yeast. From the total list of 5188 validated open reading frames of the $S$. cerevisiae genome (Fig. 4A)(Cherry et al., 2012), we initially selected four main classes of biosynthetic reactions (aiming to limit growth) that are not part of central carbon metabolism, namely 1) nucleotide metabolism, 2) cofactor/vitamin metabolism, 3) lipid metabolism, and 4) amino acid metabolism, containing a total of 325 unique genes (Fig. 4A, Supplementary Table S5). This list was further refined to focus on 3-4 selected metabolic pathways from each of the four classes (e.g. cysteine biosynthesis from homocysteine) totalling 110 unique genes, of which we omitted metabolic reactions catalysed by multiple gene products (e. g. ADE5/ADE7), bringing the gene list to 68 candidates (Fig. 4A). Of special attention, it should be noted that for selection of genes involved in amino acid biosynthesis, we initially made a complete list of the abundance of amino acids in the heterologous proteins of the VG pathway and compared this to the average amino acid composition of yeast biomass (Lange and Heijnen, 2001). For example, glutamine accounts for only $3.98 \%$ of the amino acids in the VG pathway, whereas leucine is the most abundant accounting for $10.71 \%$ (Supplementary Table S6). For comparison, the same analysis for the average composition of yeast biomass, revealed that glutamine accounts for $7.75 \%$ of yeast biomass, and leucine for $8.03 \%$ (Supplementary Table S6), from which we hypothesized that a possible limitation in glutamine biosynthesis could effectively limit growth while having minimal impact on VG productivity. Finally, we decided to include $A R O 2$ as a candidate gene since it is involved downstream of the shikimate pathway (Gottardi et al., 2017), and for which limited expression could therefore help to accumulate more shikimate intermediate and thus boost VG pathway flux. To further refine the selection criteria to an operational number for control circuit testing, we focused our attention on 56 genes described as essential or causing auxotrophy (Cherry et al., 2012), and then only selected genes with an average expression level higher than the estimated VanO-containing TEF1 promoter in the absence of VAC (TEF1_OFF) across a wide range of yeast growth rates (i.e. $0.02-0.33 \mathrm{~h}^{-1}$ ) in glucose-limited aerobic chemostats (Regenberg et al., 2006). The TEF1_OFF expression level was estimated by comparing the fluorescence intensity of the vanillic acid biosensor system in the absence of the inducer to the native TEF1 promoter (Supplementary Fig. S5). We then estimated the TEF1_ON value based on the biosensor dynamic range (Supplementary Fig. S4). Because of the low OFF state and dynamic range previously reported for the PcaQ biosensor design (Ambri et al., 2020), essential gene candidates with average expression within the VanR dynamic output range were also considered relevant for PAC control circuits founded on PcaQ. Finally, from this list of 34 genes, we manually selected 10 genes, where either accumulation or depletion of its respective substrate and product due to altered expression levels would not cause toxic effects (e.g. increased mutagenesis), as our candidate list of essential genes for control circuit testing (Fig. 4A and B).

To test the performance of the control circuits, we initially introduced the VanR biosensor design in a prototrophic CEN.PK113-7D background strain by individually replacing the genomic locus, containing the first 150bp upstream of the first ATG of each of the 10 selected essential genes, with the synthetic construct (Supplementary Fig. S6A). A similar approach was adopted for PAC control circuits, with the exception that PcaQ and the synthetic PcaO-containing CYC1 promoter were introduced into both the parental VG producing strain and the non-productive VG-3DSD $\Delta$ strains (Supplementary Fig. S6B). Following successful CRISPR-mediated promoter replacements, the
A

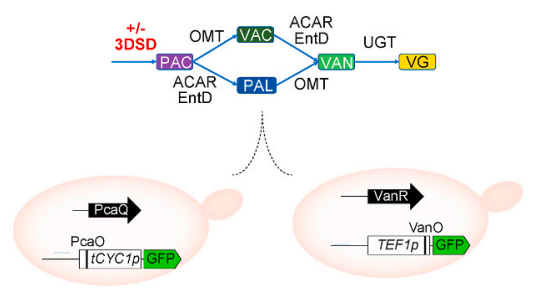

B

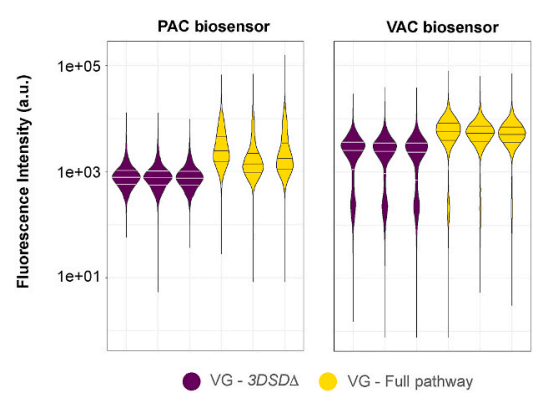

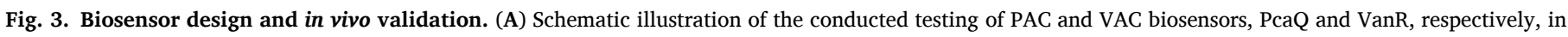

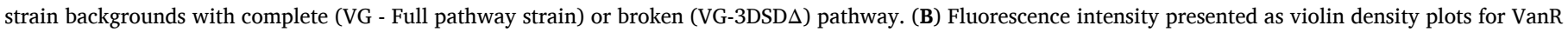
and PcaQ biosensor designs in the vanillin- $\beta$-glucoside producing strains (VG-Full pathway) and non-producing (VG-3DSD $\Delta$ ) strains. 
A

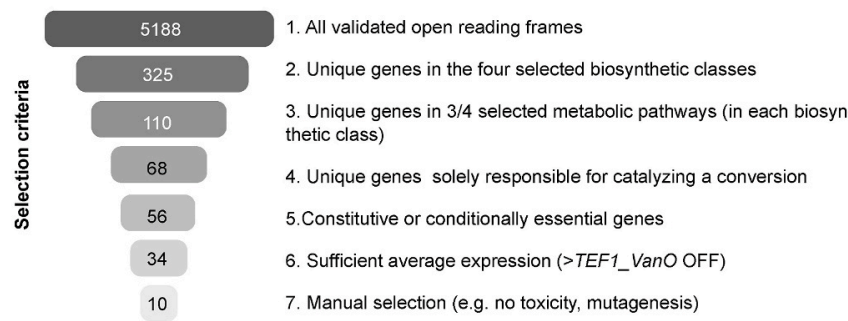

B

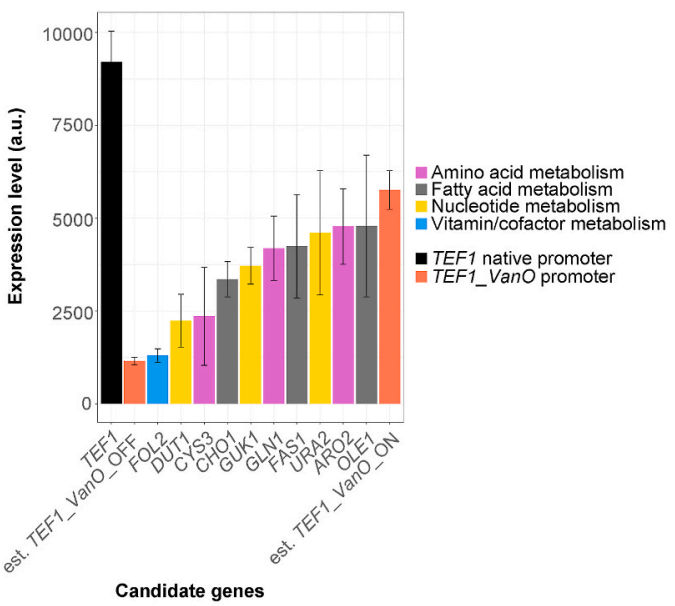

C

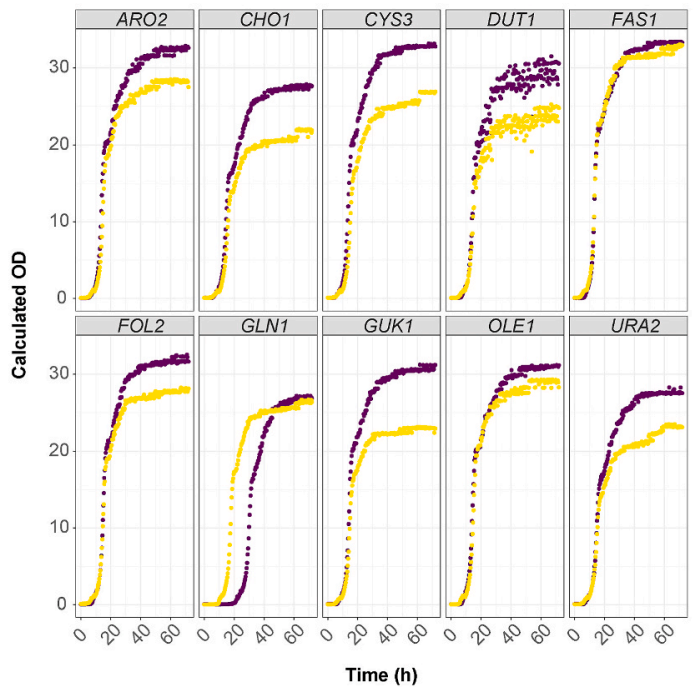

D

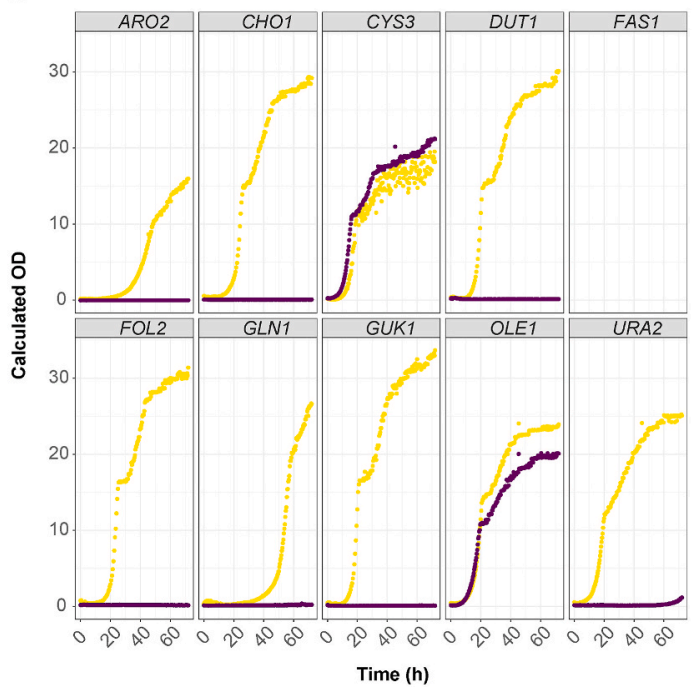

Fig. 4. Selection of essential genes and testing of VAC and PAC control circuits. (A) Schematic illustration of the various filtering criteria applied for essential gene selection. The number of genes passing each of the seven filtering steps are indicated in the grey-coloured bars, and the steps filtering criteria indicated to the right. (B) Native expression levels of essential genes selected for promoter replacement. Genes were selected for different metabolic functions: nucleotide metabolism (yellow), fatty acid metabolism (black), amino acid metabolism (purple) and vitamin/cofactor metabolism (blue). (C) Growth profile of wild type CEN.PK strains carrying the VAC control circuit design in the presence (yellow) or absence (purple) of $2 \mathrm{mM}$ vanillic acid. (D) Growth profile of VG pathway strains (yellow) and VG3DSD $\Delta$ strains (purple) carrying the PAC control circuit design. Each curve is representative of a minimum of two biological replicates. It was not possible to identify a colony having the FAS1 promoter replaced by the PAC control circuit. (For interpretation of the references to colour in this figure legend, the reader is referred to the Web version of this article.)

strains were tested for conditional or improved growth upon external feeding of VAC or internal formation of PAC in VG-producing strains compared to VG-3DSD $\Delta$ strains for the VAC and PAC control circuits, respectively.

For strains expressing the VAC control circuits, cells were grown in $\mathrm{SM}$ for $24 \mathrm{~h}$, then diluted into fresh medium in the presence or absence of VAC, and growth was subsequently monitored for $72 \mathrm{~h}$. From this, it was evident that when coupled to the glutamine synthase encoded by GLN1, the VAC control circuit enabled shortening of the initial lag phase by approx. $10 \mathrm{~h}$ in the presence of VAC, while the specific growth rate was not significantly altered between the different conditions (Fig. 4C, Supplementary Fig. S7). For the other 9 tested genes no VAC-dependent growth effects were observed.

Next, we assessed the growth when PcaQ was used to control the expression of the same set of 10 essential genes expressed from the PcaOcontaining truncated CYC1 promoter (Fig. 4D, Supplementary Fig. S8). Here, with the exception of OLE1 and CYS3, and to a lesser extent also URA2, all control circuits had completely abolished growth of nonproducing VG-3DSD $\Delta$ strains compared to VG-producing strains over
$72 \mathrm{~h}$ of cultivation (Fig. 4D).

Taken together, this demonstrates that metabolic pathways involved in four different metabolic functions can be used for designing control circuits, and suggests that the low OFF state supported by the PcaOcontaining truncated $C Y C 1$ enables a broader range of essential genes covering a larger basal expression amplitude to be used, compared to control circuits founded on the repressor-type transcriptional regulator VanR controlling the synthetic VanO-containing TEF1 promoter.

\subsection{Production stability of strains expressing control circuits}

By design, control circuits founded on genetically-encoded biosensors controlling the expression of essential genes may themselves be pressure points for fitness-burdened cells to escape heterologous anabolic product formation. In order to investigate the ability of the engineered control circuits for stably maintaining product formation over long cultivation regimes, we next introduced the VAC and PAC control circuit in the VG production strain, and repeated the batch cultivation experiment with seven sequential passages, spanning a total 
of approx. 55 generations (Fig. 2A). Again, after each transfer the extracellular concentration of metabolites was assessed by HPLC.

As determined by HPLC analysis, the parental VG strain maintained its productive lifespan for VG and all VG-pathway intermediates for one passage following the selective preculture (approx. 14 generations), yet with a $>91 \%$ loss in VG productivity following four batch transfers, and a $98.4 \%$ loss of VG productivity by the end of the sequential passage experiment (Figs. 2C and 5A). In comparison, for the strains armed with VAC control circuits one culture showed a gradual decrease in VG productivity, yet maintaining approx. 70\% VG productivity at the end of the experiment $(0.73 \mathrm{mM}-0.52 \mathrm{mM} \mathrm{VG})$, whereas the second culture had completely lost VG production by the end of the experiment (Fig. 5B), indicating that the fitness-burdened VG producing strain can still escape the VAC control circuit and/or mutate 3DSD.

For strains expressing the PAC control circuit, we repeatedly observed $>50 \%$ decrease in PAC and VG productivity from the selective preculture to transfer 1 (Fig. $5 \mathrm{C}$ ). Yet following the first transfer, productivity increased immediately during the following batch cultivations, ending the passage experiment with an increase in VG productivity of $54.5 \%$ and $72.2 \%$ for the two biological replicates, respectively (Fig. 5C). Furthermore, all the VG intermediates, with the exception of PAL, were produced at higher concentrations by the end of the passage experiment. More precisely, both biological replicates presented an approx. 30\% increase in PAC levels and approximately twice as much VAN compared to the first transfer. However, the biggest increase was observed for VAC where the two biological replicates increased productivity by $188 \%$ and $137 \%$, respectively (Fig. 5C).

Taken together, this time-resolved experiment demonstrates that control circuits coupling essential GLN1 expression with accumulation of VAC and PAC, founded on the VanR transcriptional repressor and the transcriptional activator PcaQ, respectively, can enable extension of the productive lifespan of VG pathway, and in the case of PAC control circuit both pathway intermediates and final VG product formation is increased when compared to the non-stabilized VG strain.

\subsection{Population-level sequencing to assess integrity of control circuits}

Based on the results obtained from the batch cultivations of cells with and without control circuits (Figs. 2 and 5), we next sought to determine whether i) the VanR-based control circuit mutated together with the VG pathway, and ii) if the cells expressing the PcaQ-based control circuits included mutations required to restore the growth rate and product formation following the first transfer (Fig. 5). For this purpose, we whole-genome sequenced populations following the fourth and the seventh sequential culture for strains carrying the VanR-based control circuit and the third and the seventh culture for the strains with the PcaQ-based control of GLN1 expression and we compared the results with their corresponding parental strains.

We mapped the whole-genome sequencing reads against the CEN. PK113-7D genome as a reference to assess genome coverage, basecalling and mutations. First, for the cultivation of the strains expressing the VAC control circuit, the replicate maintaining approx. $70 \%$ VG productivity following transfer 4 had no mutations identified, yet at transfer 7, a mutation localized in the VanO-containing TEF1 promoter, driving the expression of the $3 D S D$ gene, was identified (Fig. 6A). This mutation was observed in $23 \%$ of the reads from this population and localized 18 bases downstream of the transcription start site of the TEF1 promoter (Fig. 6A). Moreover, in this replicate culture, two additional nonsynonymous mutations, encoding Q91P and P208S, were identified in $13 \%$ and $15 \%$ of the reads, respectively (Supplementary Figs. S9A-B). For the second replicate culture expressing the VAC control circuit, with a complete loss of VG productivity by the end of the cultivation, a single non-synonymous mutation in 3DSD, encoding P208R, was observed in $63 \%$ of the reads by the end of the fourth culture (Fig. 6B). Furthermore, approx. $40 \%$ of the reads showed a targeted $30 \mathrm{bp}$ deletion in the TEF1 promoter exactly covering the VanO sequence (Fig. $6 \mathrm{C}$ ). For the same replicate, at transfer 7 where no VG production was observed (Fig. 5B), the P208R mutation in the coding region of the 3DSD gene was present in $98 \%$ of the reads, and reads carrying the VanO site deletion increased to approx. $65 \%$ of the total population (Fig. $6 \mathrm{~B}$ and C). Finally, from the
A

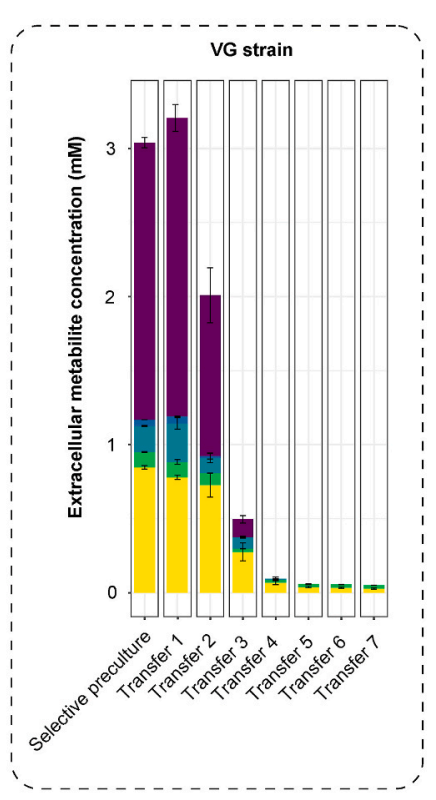

B

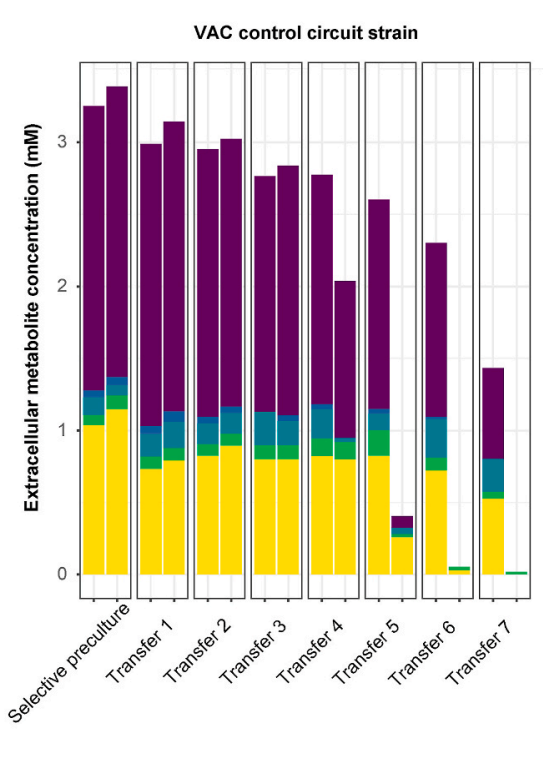

C

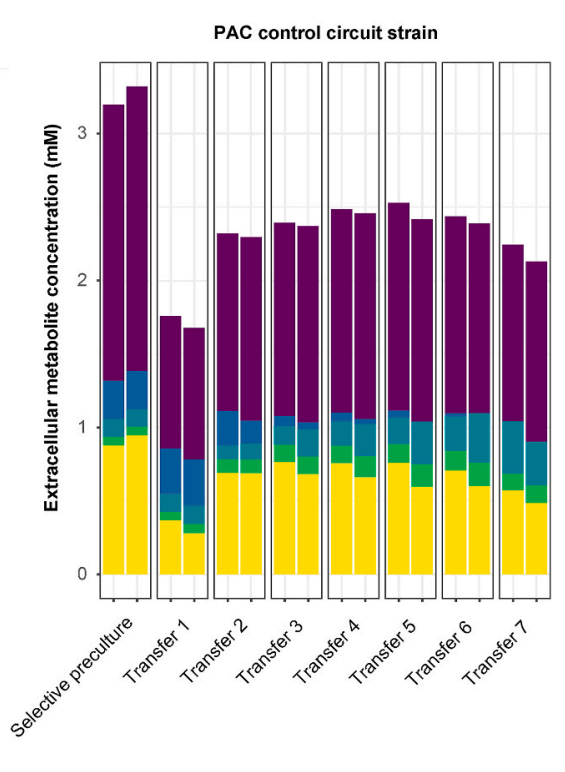

pac Pal vac van vg

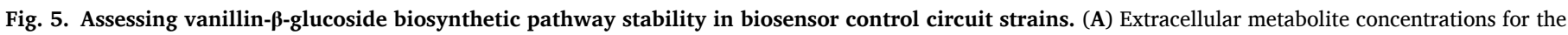

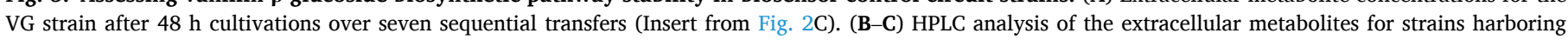

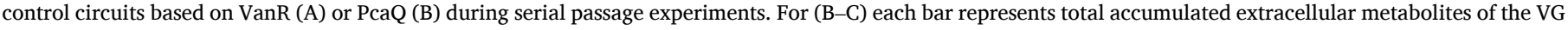
biosynthetic pathway based on one $(n=1)$ biological replicate. For $(B-C)$ each bar represents one biological replicate. 


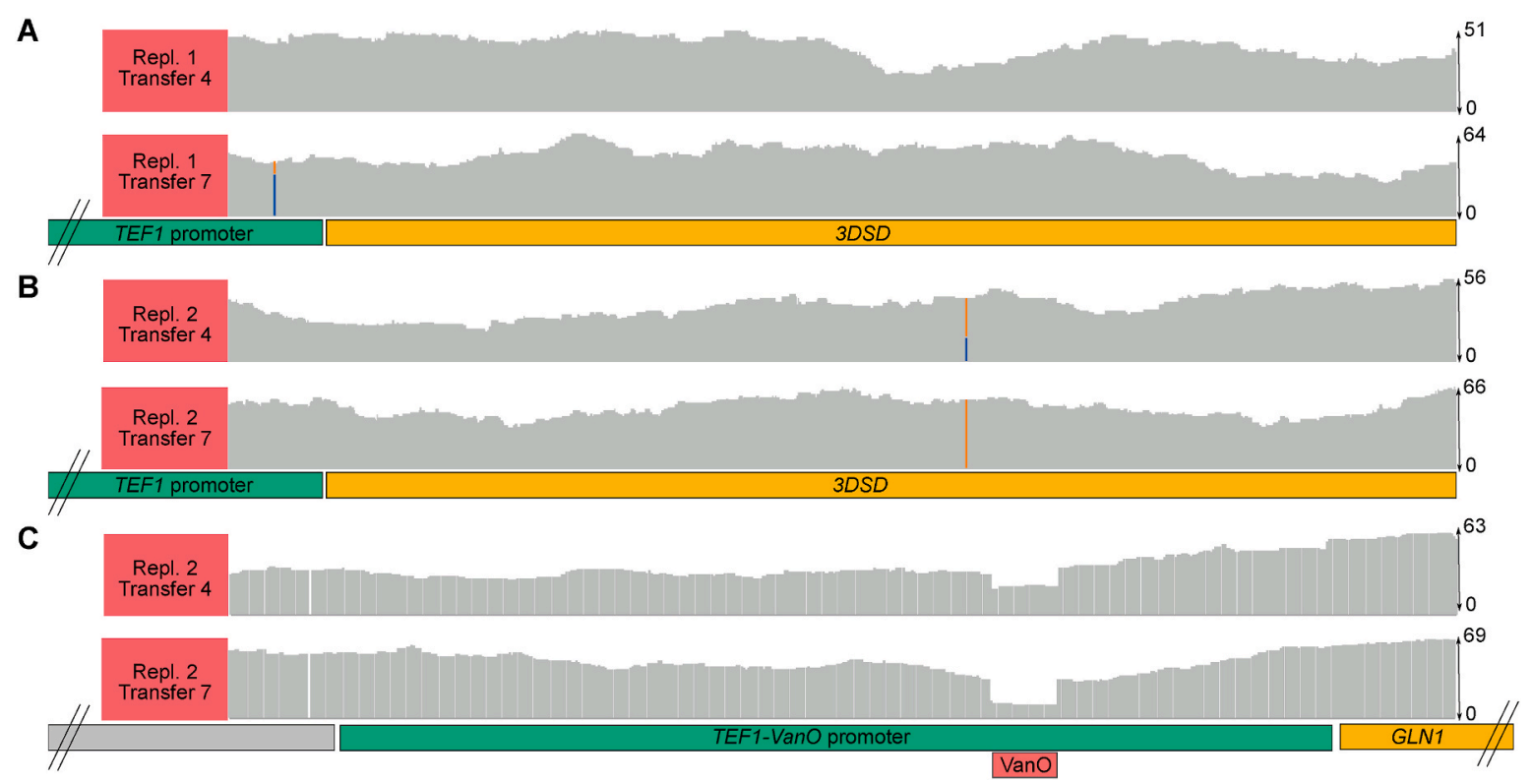

Fig. 6. Whole-genome population level sequencing identifies the error-modes of VAC control circuits. (A-B) Genome-viewer zooms on the 3DSD gene of the two replicate VAC control circuit strains at transfer 4 and 7 (see also Fig. 5A). The blue and orange bars represent the ratio of wild-type (blue) and mutated (orange) sequence reads aligned to $3 D S D$. Below the bar plots for read coverage, the genomic layout of the $3 D S D$ gene expression unit is indicated with the TEF1 presented in green and the 3DSD gene in yellow. (C) Genome-viewer zoom on the synthetic VanO-containing TEF1 promoter of the VAC control circuit strain (replicate 2, Fig. 5A). The VanO operator site is marked with a red box. Below the bar plot for read coverage, the genomic layout of the GLN1 gene expression unit is indicated with the TEF1-VanO promoter presented in green and the GLN1 gene in yellow. For (A-C) read coverage assembled to the CEN.PK113-7D reference genome is indicated to the right. (For interpretation of the references to colour in this figure legend, the reader is referred to the Web version of this article.)

whole-genome sequence analysis of the PcaQ-stabilized strains no mutations were observed in the $3 D S D$ gene or the PcaQ biosensor design (Supplementary Fig. S10).

Taken together, these results show that, at population level, both the VAC control circuit and $3 D S D$ are mutated for yeast to escape fitnessburdened VG production. Furthermore, for the strains expressing the VAC control circuit, these results suggest that the $3 D S D$ gene is likely to mutate before the synthetic TEF1 promoter controlling the expression of the GLN1 gene. On the contrary, strains expressing the PAC control circuit, founded on the transcriptional activator PcaQ and the minimalized synthetic CYC1 promoter, enables robust expansion of the productive lifespan of the VG pathway, without any observed genetic perturbations.

\subsection{Laboratory-scale fed-batch process}

Based on the successful application of the control circuits for coupling VG pathway stability to essential glutamine biosynthesis via the GLN1 gene during sequential shake flask experiments, we tested the VAC and PAC control circuit designs in a fed-batch process in order to test the control circuit under conditions often applied in industry. First, to mimic the preceding seed train, we grew pre-cultures of strains with
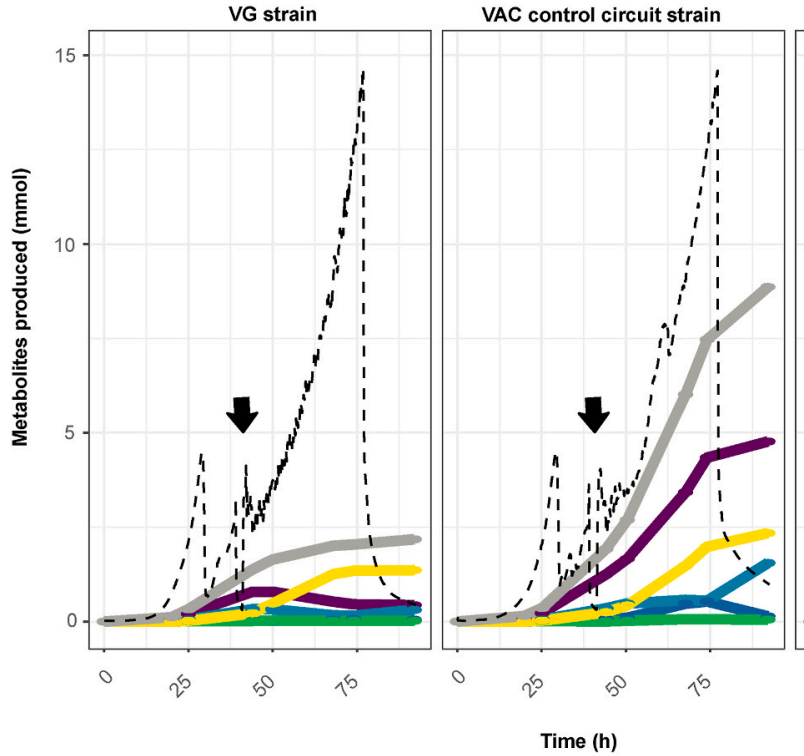

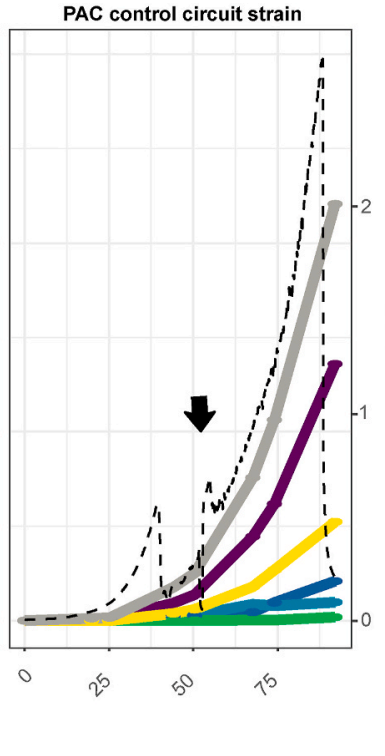

Fig. 7. Fed-batch bioprocess for parental VG strain and strains expressing VAC and PAC control circuits. Extracellular metabolite and growth characterization of the parental VG strain (left panel), and strains expressing VAC (middle panel) and PAC (right panel) control circuits during the batch and fed-batch bioprocess. Metabolite production is expressed in mmol of VG pathway metabolites produced during the cultivation. The values were corrected for the volume of medium removed for HPLC sampling and represent one $(n=1)$ biological replicate. Data for a second biological replicate can be found in Supplementary Fig. S12. Dashed black lines represent off-gas $\mathrm{CO}_{2}$ (percent), while black arrows represent the beginning of the fed-batch phase as inferred from initial increase in dissolved oxygen (see also Supplementary Fig. S11). Coloured lines indicate accumulation of individual VG pathway metabolites during the cultivation (Time, h) according to colour coding indicated at the bottom of the plot. (For interpretation of the references to colour in this figure legend, the reader is referred to the Web version of this article.) 
and without the VAC or PAC control circuit under non-selective conditions in rich medium. Following $24 \mathrm{~h}$, the pre-cultures were used to inoculate the first seed-train culture in a culture tube with a 1:100 inoculation ratio. Following another $48 \mathrm{~h}, 40 \mu \mathrm{L}$ of this first seed-train culture was transferred to a 4-mL shake flask culture, and after another $48 \mathrm{~h}$ this culture was used to inoculate a $400-\mathrm{mL}$ bioreactor. This seed train from $40 \mu \mathrm{L}$ to $400 \mathrm{~mL}$ represents the same number of generations encountered in industrial seed trains starting from $1 \mathrm{~L}$ and ending at 10,000 L (Fu et al., 2014).

The bioreactor culture started with a batch phase using $20 \mathrm{~g} / \mathrm{L}$ of glucose and after carbon depletion, indicated by a rapid rise in dissolved oxygen levels and drop of off-gas $\mathrm{CO}_{2}$, the fed-batch phase was started (Fig. 7, and Supplementary Figs. S11A-C). During the fed-batch, a concentrated solution of feed medium was used, and the growth rate was set to $0.05 \mathrm{~h}^{-1}$ (Supplementary Table S7). Samples were taken at defined time-points $(0,20,25,44,50,68,74$ and $92 \mathrm{~h})$ during the batch and fedbatch phases, and metabolite consumption and formation was determined off-line via HPLC analysis (Fig. 7, Supplementary Fig. S12). The cultures were stopped when the working volume in the bioreactor reached $900 \mathrm{~mL}$. For the parental VG strain, the batch phase stopped after $40 \mathrm{~h}$, during which approx. $1.4 \mathrm{mmol}$ of VG pathway metabolites were formed from the initial $20 \mathrm{~g} / \mathrm{L}$ of glucose (Fig. 7). Similarly, the batch phase of the strain expressing VAC control circuit terminated after $40 \mathrm{~h}$, yet with $1.9 \mathrm{mmol}$ of VG pathway metabolites accumulated (Fig. 7). In contrast, the strain expressing the PAC control circuit grew slower, and the batch phase only finished after $55 \mathrm{~h}$, during which approx. $1.3 \mathrm{mmol}$ VG pathway metabolites accumulated (Fig. 7). These results are in agreement with the observed drop in productivity of the parental VG strain after the second transfer in the sequential batch culture experiment (Fig. 2B), suggesting that a fraction of parental VG producing cells in the inoculum of the bioreactor already exhibited loss of productivity. Following the initial batch phase, the impact of the control circuits became even more apparent in the fed-batch phase. Here, the strain expressing the PAC control circuit outperformed both the parental VG strain and the strain expressing the VAC control circuit by producing $11 \mathrm{mmol}$ of VG pathway metabolites compared to approx. $2.2 \mathrm{mmol}$ and $8.9 \mathrm{mmol}$ of VG pathway metabolites for the VG and VAC control circuit strains, respectively (Fig. 7). Importantly, even though the strains expressing control circuits accumulate 4-5-fold higher amounts of total VG pathway metabolites compared to the parental VG strain, these strains also accumulate higher amounts of the VG endproduct. Indeed, for the strains expressing the VAC and PAC control circuits, at the end of the fermentation, a total of $2.34 \mathrm{mmol}$ and 2.61 $\mathrm{mmol}$ of VG, respectively, is produced, compared to $1.36 \mathrm{mmol}$ of VG produced by the parental VG strain, representing up to almost 2-fold improvement in production (Fig. 7). However, for the strains expressing the control circuits, there is also a substantial increase in the accumulation of VG pathway intermediates. For instance, and as also expected for control circuits founded on a PAC biosensor, in the strain expressing the PAC control circuit $6.8 \mathrm{mmol}$ of PAC is accumulated, which is approx. 15 times the amount produced by the parental VG strain, and $\sim 40 \%$ more compared to the VAC control circuit strain. Likewise, the strain expressing the VAC control circuit accumulates more VAC ( $1.5 \mathrm{mmol})$ than both the parental VG strain and the PAC control circuit strain (0.3 mmol and $0.5 \mathrm{mmol}$, respectively) (Fig. 7). Furthermore, in the parental VG strains PAC accumulates until the measurement at $50 \mathrm{~h}$, following which the PAC levels drastically decrease, corroborating the findings from the batch cultivations (Fig. 2). Similarly, PAL, which is almost entirely converted to VAN in the parental VG strains, accumulates to 0.14 and $1.05 \mathrm{mmol}$ at the end of the cultivation in the strains expressing the VAC and PAC control circuit, while for VAN the parental VG strain accumulate $0.02 \mathrm{mmol}$ compared to 0.05 and $0.09 \mathrm{mmol}$ for the strains expressing the VAC and PAC control circuits, respectively (Fig. 7).

In summary, the mimic of an industrial scale-up process, validates the use of control circuits founded on biosensors for biosynthetic pathway intermediates coupled to expression of essential genes, to prolong and improve the productive lifespan of yeast cell factories for anabolic end-product formation. Having said this, it is evident that PAC accumulated to relative high levels. Here it should be noted that in batch cultures, VG producing strains have been described to first grow respirofermentative, producing ethanol and predominantly PAC, and only when glucose is depleted, switch to fully respiratory growth on ethanol from which predominantly VG is formed (Strucko et al., 2015). In our study, the carbon/glucose-limited fed-batch was indeed operated below the growth rate at which S. cerevisiae switches to respiro-fermentative growth (Chen and Nielsen, 2019), which thus could explain the relatively high levels of PAC.

\section{Discussion}

Engineering microorganisms to produce valuable chemicals and fuels has the potential to enable the transition towards a greener and more sustainable bio-based economy (Nielsen and Keasling, 2016). However, the metabolic burden caused by engineering heterologous pathways for anabolic product formation in cell factories is often associated with reduced fitness and growth rate (Strucko et al., 2015; Wu et al., 2016). This ultimately imposes a selective pressure on engineered cells to reroute nutrients away from burdening non-essential pathways, or to delete these pathways completely, towards maximising cell proliferation (Rugbjerg et al., 2018a, 2018b).

Based on the result of the sequential shake flasks experiments we determined that strains expressing control circuits outperformed the parental strain without a control circuit, as strains expressing control circuits enabled strains to remain productive for $>55$ generations in batch cultivations, compared to merely 25 generations observed for the parental strain (Figs. 2 and 5). Moreover, comparing the performance of these strains in a fed-batch cultivation mimicking industrial setups, strains expressing control circuits accumulated up to 5-fold higher amounts of total pathway metabolites, including up to 2-fold higher endproduct formation for VAC and PAC control circuits, respectively. This demonstrates that biosensors derived from prokaryotic small-molecule binding transcriptional regulators, can be successfully employed in yeast to expand the productive lifespan and control evolutionary drift of fitness-burdened cell factories, even when coupling essential gene expression with formation of product pathway intermediates.

Moreover, this study highlights how physiological, genetic, and bioinformatic approaches enable the engineering of control circuits founded on pathway intermediates. Specifically, by constructing single knockout gene deletions for all heterologous pathway steps, we were able to identify the key fitness-burdening pressure point in strains expressing the VG pathway. Next, by mining publicly available transcriptome and phenome datasets (Cherry et al., 2012; Regenberg et al., 2006), this study demonstrates successful selection criteria for identification of candidate essential genes, encoding a broad array of metabolic functions, which can be coupled to biosensors and thus enable selective growth based on small-molecule concentrations. With the increasing number of biosensors being developed, and demonstrations of fermentation-based manufacturing of valuable chemicals based on anabolic metabolism (Koch et al., 2019; Nielsen and Keasling, 2016), this combined approach should be applicable for stabilizing and optimizing many more bioprocesses in the future. Specifically, in this study, the stability of a fitness-burdened yeast cell factory was characterised and compared with strains expressing two different control circuit designs, capable of detecting pathway intermediates, coupled to essential gene expression. By focusing on biosensing of intermediates, our goal was to provide experimental guidelines to assess whether already established biosensors for pathway intermediates could be employed to successfully extend the productivity lifespan of a strain, without the need to engineer new biosensors for specific end-products. Moreover, by developing detecting intermediates it should be possible to re-use the same biosensor design to stabilize the production of other pathways 
relying on the same intermediate, such as biosynthesis of cis, cis-muconic acid (Weber et al., 2012).

Having said this, this study not only exemplifies the error-modes of a fitness-burdening heterologous biosynthetic pathway, but also elucidates those observed in genetically-encoded control circuits, as those adopted in this study. First, by employing both transcriptional activators and repressors we observed that the PcaQ activator allowed for a wider range of essential genes to be used (Fig. 4). Here, when replacing the native promoters with the PcaO-containing minimal CYC1 promoter controlled by PcaQ, most of the tested essential genes were only able to sustain growth in strains producing PAC. On the contrary, when VanR was employed, all tested genes, with the exception of GLN1, showed similar growth upon VAC supplementation compared to control conditions. This is likely due to the lower OFF state of essential gene expression provided by the PAC control circuit founded on the minimal PcaO-containing CYC1 promoter, highlighting the importance of biosensor output tuning when coupled to growth-based selection. Moreover, since many repressor-type biosensors established in $S$. cerevisiae rely on reporters with strong promoters to provide a readable output (Ambri et al., 2020; Dabirian et al., 2019; David et al., 2016; Hector and Mertens, 2017), this issue is believed to be a common concern for future strain stability efforts employing transcriptional repressors. Indeed, even though both types of control circuits lead to an increase in transcription in the presence of the ligand, inactivating mutations in the control circuits will lead to different outcomes depending on the aTF mode-of-action (D'Ambrosio and Jensen, 2017). With a repressor-based control circuit, any mutation limiting DNA-binding affinity will lock the expression of the actuating gene (e.g. an essential gene) in a quasi-ON state, just as deletion of non-native aTF repressor binding sites in output promoter is likely to do (Ambri et al., 2020). This quasi-ON state indeed proved to be sufficient for GLN1 in this study (Figs. 5B and 6C). Contrastingly, if an activator-type aTF is mutated, the system will be locked in an OFF state, maintaining non-producing cells in a low-fitness stage. Thus, we recommend designs of control circuits based on short sequence-diverse synthetic promoters (Kotopka and Smolke, 2019; Redden and Alper, 2015) and activator-type aTFs to limit the rapid spreading of simple error-modes towards quasi-ON expression of output genes or homologous recombination events as observed for the "sniper-attack" on 30 bp VanO in the TEF1-based output promoter (Fig. 6C).

Still, with meticulous characterization of the error-modes of cell factories and the input-output relationship connecting aTF biosensors with essential metabolic functions, control circuits are transforming biosensors from mere high-throughput screening technologies into an integral part of stabilizing bioprocesses, as already exemplified for bacterial cell factories (Rugbjerg et al., 2018b; Wang and Dunlop, 2019; Xiao et al., 2016). Ultimately, this will also enable understanding of the underlying mechanisms controlling population heterogeneity, and significantly contribute to development of new robust and cost-effective bio-based production processes, including long-term performance (500-1000 generations) making biomass reuse an option and reducing the need for seed trains. Beyond metabolic engineering, the engineering of control circuits also should hold potential for successful applications within other disciplines, including advanced microbiome therapeutics.

\section{Methods}

\subsection{Cultivation media and conditions}

Chemically competent Escherichia coli DH5 $\alpha$ strain was used as a host for cloning and plasmid propagation. The cells were cultivated at $37^{\circ} \mathrm{C}$ in 2xYT supplemented with $100 \mu \mathrm{g} / \mathrm{mL}$ ampicillin. The Saccharomyces cerevisiae strains used in this study were grown at $30{ }^{\circ} \mathrm{C}$ and $250 \mathrm{rpm}$ in three types of media: yeast extract peptone (YP) medium (10 g/L Bacto yeast extract and $10 \mathrm{~g} / \mathrm{L}$ Bacto peptone), synthetic complete (SC) medium $(6.7 \mathrm{~g} / \mathrm{L}$ yeast nitrogen base without amino acids with appropriate drop-out medium supplement) and synthetic medium (SM) prepared as previously described (Mans et al., 2018). All three media were supplemented with glucose $20 \mathrm{~g} / \mathrm{L}$ as carbon source unless otherwise specified. For media preparation, salts and water were sterilised in the autoclave at $120^{\circ} \mathrm{C}$, before the complete mixed media were sterile filtered with 0.2 $\mu \mathrm{m}$ filters.

\subsection{Plasmids and strains construction}

All plasmids used in this study were assembled by USER ${ }^{\mathrm{TM}}$ (uracilspecific excision reagent) cloning (New England Biolabs). Biobricks constituting promoters, genes and other genetic elements required to assemble the plasmids were amplified by PCR using PhusionU polymerase (Thermo Fisher Scientific). S. cerevisiae strains were constructed by the lithium acetate/single-stranded carrier DNA/PEG method previously described (Gietz and Schiestl, 2007). The complete list of strains and plasmids used in this study is provided in Supplementary Tables S1 and S2.

\subsection{HPLC detection of extracellular metabolites}

All metabolites of the vanillin $\beta$-glucoside pathway were analysed by HPLC using the Dionex Ultimate 3000 HPLC (Thermo Fisher Scientific), coupled with the Supelco Discovery HS F5-3 HPLC column $(150 \times 2.1$ $\mathrm{mm} \times 3 \mu \mathrm{m}$ ) (Sigma Aldrich). Mobile phase A consisted of $10 \mathrm{mM}$ ammonium formate, $\mathrm{pH} 3$ while mobile phase B consisted of acetonitrile. The elution profile was as follows: $5 \%$ of solvent $\mathrm{B}$ for $0.5 \mathrm{~min}$ and increased linearly to $60 \%$ B over $5 \mathrm{~min}$. The gradient was increased to $90 \%$ B over $0.5 \mathrm{~min}$ and kept at this condition for $2 \mathrm{~min}$. Finally, returned to $5 \% \mathrm{~B}$ and equilibrated until $10 \mathrm{~min}$. The flow rate was set at $0.7 \mathrm{~mL} / \mathrm{min}$ while the column was held at $30{ }^{\circ} \mathrm{C}$ and the metabolites were detected using the UV diodide detector DAD-3000 Diode Array Detector set at 260, 277, 304 and $210 \mathrm{~nm}$. The samples were prepared as previously described (Strucko et al., 2017). Shortly, $1 \mathrm{~mL}$ of yeast culture and $1 \mathrm{~mL}$ of $96 \%$ ethanol were mixed. The solution was then centrifuged at $12000 \mathrm{~g}$ for $2 \mathrm{~min}$. The supernatant was then collected and stored at $-20{ }^{\circ} \mathrm{C}$ until it was measured on the HPLC.

\subsection{Biosensor design and promoter replacement}

The bidirectional VanR design used to replace essential genes promoters is composed of the $A D H 1$ terminator, VanR from Caulobacter crescentus, the PGK1 promoter driving the expression of VanR and the engineered TEF1p containing two VanO sequences separated by the Eco47III restriction site. The design as described was used to replace the $150 \mathrm{bp}$ sequence upstream of the selected essential genes. The PcaQ biosensor design is composed of two parts: PcaQ, under the control of the strong TDH3 promoter and the reporter module composed of the truncated CYC1 promoter containing the PcaQ binding site, both previously described (Ambri et al., 2020). The complete list of the gRNAs used in this study can be found in Supplementary Table S3. Each gRNA sequence was then combined with a backbone carrying the pRNR2-Cas9-CYC1t cassette to assemble an all-in-one CRISPR plasmid, by USERTM (uracil-specific excision reagent) cloning (New England Biolabs), as previously described (D'Ambrosio et al., 2020). In order to maximise the transformation efficiency the plasmid were used to transform yeast strains where one additional copy of TEF1p-Cas9-CYC1t was already present in the genome within the EasyClone site X-4 site (Jensen et al., 2014).

\section{Growth profiler analysis}

\subsection{Cell growth assessment}

Cell growth was evaluated using the Growth Profiler 960 (Enzyscreen B.V., The Netherlands) at $30^{\circ} \mathrm{C}$ and $200 \mathrm{rpm}$ or the ELx808 
Absorbance Microplate Reader (BioTek) at $30{ }^{\circ} \mathrm{C}$ and "fast" shaking. Prior to Growth Profiler measurement, $S$. cerevisiae strains, with the exception of strains carrying the PcaQ biosensor design, were inoculated in $0.5 \mathrm{~mL}$ of synthetic medium in 96 -format polypropylene deep-well plates, and grown overnight at $30{ }^{\circ} \mathrm{C}$ and $300 \mathrm{rpm}$. The cells were then diluted 1:100 in fresh synthetic medium. Because of the longer lag phase or inability to grow in SM medium (Supplementary Fig. S8), the strains where the native essential gene promoter was replaced with the PcaQ biosensor design were initially inoculated in rich YPD medium and grown overnight. Next, the strains were diluted 1:10 in synthetic medium and cultured overnight. Finally, the strains were diluted 1:100 in fresh synthetic medium to a final volume of $150 \mu \mathrm{L}$ and the cell suspension was transferred to a 96-half deepwell plate (Enzyscreen B.V., The Netherlands). In order to convert the G-values provided by the instrument into $\mathrm{OD}_{600}$ values we generated a calibration curve by measuring the G-value of samples with known OD. The strains measured by the ELx 808 were cultivated in the same manner, and the $\mathrm{OD}_{630}$ values were used to assess the growth.

\subsection{Flow cytometry measurements}

Single cell fluorescence was evaluated using a Becton Dickinson LSR FORTESSA equipped with a blue $488 \mathrm{~nm}$ laser. Prior to measurement, S. cerevisiae strains were grown overnight in mineral medium at $30{ }^{\circ} \mathrm{C}$ and $300 \mathrm{rpm}$ in 96-format polypropylene deep-well plates. Next, the cells were diluted 1:50 in fresh mineral medium in the presence or absence of the inducer and were cultivated for $20 \mathrm{~h}$. Finally, cells were diluted 1:20 in PBS to arrest cell growth and the fluorescence was measured by flow cytometry. For each sample 10,000 single-cell events were recorded.

\subsection{Whole genome sequencing}

The samples selected for whole genome sequence were inoculated from glycerol stock in rich YPD medium and cultured overnight. The genomic DNA was extracted by using the Quick-DNA Fungal/Bacterial Miniprep Kit (Zymoresearch) following the provided protocol. DNA libraries were then prepared using a Kapa Hyper Prep Library Prep Kit (Roche) and sequenced by Illumina MiSeq. Data mapping was performed against the CEN.PK113-7D genome (Salazar et al., 2017) where additional expression cassettes relative to vanillin- $\beta$-glucoside biosynthetic pathway genes and control circuits were previously added. Data processing and chromosome copy number variation determinations were performed as previously described (Nijkamp et al., 2012; Verhoeven et al., 2017).

\subsection{Fed-batch cultivations}

The bioreactor cultures were performed in $1 \mathrm{~L}$ bioreactors (Biostat $\mathrm{Q}$ Plus, Sartorius, Gottingen, Germany) and started with a batch phase using $400 \mathrm{~mL}$ synthetic medium containing $20 \mathrm{~g} / \mathrm{L}$ of glucose and $5 \mathrm{~mL}$ Antifoam 204 (Sigma A6426). The temperature was maintained at $30{ }^{\circ} \mathrm{C}$ and the $\mathrm{pH}$ was kept constant at 5.0 by dropwise addition of a $10 \mathrm{M} \mathrm{KOH}$ solution. The cultures were sparged with pressurized air at a flow rate of $500 \mathrm{~mL} / \mathrm{min}$ and dissolved oxygen levels were maintained above $50 \%$ by controlling the stirrer speed. The off-gas $\mathrm{CO}_{2}$ and $\mathrm{O}_{2}$ was monitored throughout the cultivation (Prima BT Mass Spectrometer, Thermo Fisher Scientific) and the data was acquired by the Lucullus software (Securecell AG, Switzerland). After carbon depletion, indicated by a rapid rise in dissolved oxygen levels, the fed-batch phase was automatically initiated (Supplementary Fig. S9). During the fed-batch phase, a $2 x$ concentrated SM solution containing $200 \mathrm{~g} / \mathrm{L}$ glucose and Antifoam $\left(1 \mathrm{~mL} / \mathrm{L}\right.$ ), was used. The initial feed rate was set to $2 \mathrm{~mL} \mathrm{~h}^{-1}$, resulting in a growth rate of $0.05 \mathrm{~h}^{-1}$ thus ensuring an identical length of the fedbatch cultivation for all the tested strains. Throughout the fed-batch phase, the feed rate was continuously increased with a rate of 0.05 $\mathrm{h}^{-1}$ to maintain a constant growth rate. Samples were taken manually at defined time points during the batch and fed-batch phase and metabolite consumption and formation were determined via HPLC analysis.

\section{Author contributions}

RM, PR, MOAS, JDK and MKJ conceived the study. VD, ED, RDB and RM conducted all experimental work related to strain designs and construction, MvdB performed all nextgeneration sequencing analysis, while $\mathrm{JtH}$ and SS conducted all fermentation. VD, RM, and MKJ wrote the manuscript.

\section{Declaration of competing interestCOI}

JDK has a financial interest in Amyris, Lygos, Demetrix, Maple Bio, and Napigen. PR has a financial interest in Enduro Genetics ApS. MOAS has financial interest in UNION therapeutics, Biosyntia, ClinicalMicrobiomics, UTILITY therapeutics and SNIPR holding.

\section{Acknowledgements}

This work was supported by the Novo Nordisk Foundation and by the European Union's Horizon 2020 research and innovation programme under the Marie Sklodowska-Curie action PAcMEN (grant agreement No. 722287). Finally, we would like to thank Dr. Tomas Strucko for sharing with us the parental $S$. cerevisiae VG strain used in this study.

\section{Appendix A. Supplementary data}

Supplementary data to this article can be found online at https://doi. org/10.1016/j.ymben.2020.07.006.

\section{References}

Ambri, F., D’Ambrosio, V., Di Blasi, R., Maury, J., Jacobsen, S.A.B., McCloskey, D., Jensen, M.K., Keasling, J.D., 2020. High-resolution scanning of optimal biosensor reporter promoters in yeast. ACS Synth. Biol. https://doi.org/10.1021/ acssynbio.9b00333.

Brochado, A.R., Matos, C., Møller, B.L., Hansen, J., Mortensen, U.H., Patil, K.R., 2010. Improved vanillin production in baker's yeast through in silico design. Microb. Cell Factories 9, 84. https://doi.org/10.1186/1475-2859-9-84.

Carlquist, M., Fernandes, R.L., Helmark, S., Heins, A.-L., Lundin, L., Sørensen, S.J., Gernaey, K.V., Lantz, A.E., 2012. Physiological heterogeneities in microbial populations and implications for physical stress tolerance. Microb. Cell Factories 11, 94. https://doi.org/10.1186/1475-2859-11-94.

Chen, Y., Nielsen, J., 2019. Energy metabolism controls phenotypes by protein efficiency and allocation. Proc. Natl. Acad. Sci. 116 (35), 17592-17597. https://doi.org/ 10.1073/pnas.1906569116.

Cherry, J.M., Hong, E.L., Amundsen, C., Balakrishnan, R., Binkley, G., Chan, E.T., Christie, K.R., Costanzo, M.C., Dwight, S.S., Engel, S.R., Fisk, D.G., Hirschman, J.E., Hitz, B.C., Karra, K., Krieger, C.J., Miyasato, S.R., Nash, R.S., Park, J., Skrzypek, M. S., Simison, M., Weng, S., Wong, E.D., 2012. Saccharomyces Genome Database: the genomics resource of budding yeast. Nucleic Acids Res. 40 https://doi.org/10.1093/ nar/gkr1029. D700-5.

Dabirian, Y., Gonçalves Teixeira, P., Nielsen, J., Siewers, V., David, F., 2019. FadR-based biosensor-assisted screening for genes enhancing fatty Acyl-CoA pools in Saccharomyces cerevisiae. ACS Synth. Biol. 8, 1788-1800. https://doi.org/10.1021/ acssynbio.9b00118.

D’Ambrosio, V., Jensen, M.K., 2017. Lighting up yeast cell factories by transcription factor-based biosensors. FEMS Yeast Res. 17 https://doi.org/10.1093/femsyr/ fox076.

D’Ambrosio, V., Pramanik, S., Goroncy, K., Jakociunas, T., Schönauer, D., Davari, M.D., Schwaneberg, U., Keasling, J.D., Jensen, M.K., 2020. Directed evolution of VanR biosensor specificity in yeast. Biotechnol. Notes. https://doi.org/10.1016/j. biotno.2020.01.002.

David, F., Nielsen, J., Siewers, V., 2016. Flux control at the malonyl-CoA node through hierarchical dynamic pathway regulation in Saccharomyces cerevisiae. ACS Synth. Biol. 5, 224-233. https://doi.org/10.1021/acssynbio.5b00161.

Elowitz, M.B., Levine, A.J., Siggia, E.D., Swain, P.S., 2002. Stochastic gene expression in a single cell. Science 297, 1183-1186. https://doi.org/10.1126/science.1070919.

Fernandez-López, R., Ruiz, R., de la Cruz, F., Moncalián, G., 2015. Transcription factorbased biosensors enlightened by the analyte. Front. Microbiol. 6, 648. https://doi. org/10.3389/fmicb.2015.00648.

Flachbart, L.K., Sokolowsky, S., Marienhagen, J., 2019. Displaced by deceivers: prevention of biosensor cross-talk is pivotal for successful biosensor-based high- 
throughput screening campaigns. ACS Synth. Biol. 8, 1847-1857. https://doi.org/ 10.1021/acssynbio.9b00149.

Fu, Z., Verderame, T.D., Leighton, J.M., Sampey, B.P., Appelbaum, E.R., Patel, P.S., Aon, J.C., 2014. Exometabolome analysis reveals hypoxia at the up-scaling of a Saccharomyces cerevisiae high-cell density fed-batch biopharmaceutical process. Microb. Cell Factories 13, 32. https://doi.org/10.1186/1475-2859-13-32.

Gietz, R.D., Schiestl, R.H., 2007. High-efficiency yeast transformation using the LiAc/SS carrier DNA/PEG method. Nat. Protoc. 2, 31-34. https://doi.org/10.1038/ nprot.2007.13.

Gitzinger, M., Kemmer, C., Fluri, D.A., El-Baba, M.D., Weber, W., Fussenegger, M., 2012 The food additive vanillic acid controls transgene expression in mammalian cells and mice. Nucleic Acids Res. 40, e37. https://doi.org/10.1093/nar/gkr1251.

Gomes, J., Chopda, V., Rathore, A.S., 2018. Monitoring and control of bioreactor: basic concepts and recent advances. In: Komives, C., Zhou, W. (Eds.), Bioprocessing Technology for Production of Biopharmaceuticals and Bioproducts. John Wiley \& Sons, Inc., Hoboken, NJ, USA, pp. 201-237. https://doi.org/10.1002/ 9781119378341.ch6.

Gottardi, M., Reifenrath, M., Boles, E., Tripp, J., 2017. Pathway engineering for the production of heterologous aromatic chemicals and their derivatives in Saccharomyces cerevisiae: bioconversion from glucose. FEMS Yeast Res. 17 https:/ doi.org/10.1093/femsyr/fox035.

Gu, H., Zhu, Y., Peng, Y., Liang, X., Liu, X., Shao, L., Xu, Y., Xu, Z., Liu, R., Li, J., 2019 Physiological mechanism of improved tolerance of Saccharomyces cerevisiae to lignin-derived phenolic acids in lignocellulosic ethanol fermentation by short-term adaptation. Biotechnol. Biofuels 12, 268. https://doi.org/10.1186/s13068-0191610-9.

Guo, Z., Olsson, L., 2014. Physiological response of Saccharomyces cerevisiae to weak acids present in lignocellulosic hydrolysate. FEMS Yeast Res. 14, 1234-1248. https://doi.org/10.1111/1567-1364.12221.

Hansen, E.H., Møller, B.L., Kock, G.R., Bünner, C.M., Kristensen, C., Jensen, O.R., Okkels, F.T., Olsen, C.E., Motawia, M.S., Hansen, J., 2009. De novo biosynthesis of vanillin in fission yeast (Schizosaccharomyces pombe) and baker's yeast (Saccharomyces cerevisiae). Appl. Environ. Microbiol. 75, 2765-2774. https://doi. org/10.1128/AEM.02681-08.

Hector, R.E., Mertens, J.A., 2017. A synthetic hybrid promoter for xylose-regulated control of gene expression in Saccharomyces yeasts. Mol. Biotechnol. 59, 24-33. https://doi.org/10.1007/s12033-016-9991-5.

Jain, D., 2015. Allosteric control of transcription in GntR family of transcription regulators: a structural overview. IUBMB Life $67,556-563$. https://doi.org/ 10.1002/iub.1401.

Jensen, N.B., Strucko, T., Kildegaard, K.R., David, F., Maury, J., Mortensen, U.H., Forster, J., Nielsen, J., Borodina, I., 2014. EasyClone: method for iterative chromosomal integration of multiple genes in Saccharomyces cerevisiae. FEMS Yeast Res. 14, 238-248. https://doi.org/10.1111/1567-1364.12118.

Koch, M., Pandi, A., Borkowski, O., Batista, A.C., Faulon, J.-L., 2019. Custom-made transcriptional biosensors for metabolic engineering. Curr. Opin. Biotechnol. 59, 78-84. https://doi.org/10.1016/j.copbio.2019.02.016.

Kotopka, B.J., Smolke, C.D., 2019. Model-driven generation of artificial yeast promoters. bioRxiv. https://doi.org/10.1101/748616.

Kruyer, N.S., Peralta-Yahya, P., 2017. Metabolic engineering strategies to bio-adipic acid production. Curr. Opin. Biotechnol. 45, 136-143. https://doi.org/10.1016/j. copbio.2017.03.006.

Lange, H.C., Heijnen, J.J., 2001. Statistical reconciliation of the elemental and molecular biomass composition of Saccharomyces cerevisiae. Biotechnol. Bioeng. 75, 334-344. https://doi.org/10.1002/bit.10054.

Luziatelli, F., Brunetti, L., Ficca, A.G., Ruzzi, M., 2019. Maximizing the efficiency of vanillin production by biocatalyst enhancement and process optimization. Front. Bioeng. Biotechnol. 7, 279. https://doi.org/10.3389/fbioe.2019.00279.

Mans, R., Wijsman, M., Daran-Lapujade, P., Daran, J.-M., 2018. A protocol for introduction of multiple genetic modifications in Saccharomyces cerevisiae using CRISPR/Cas9. FEMS Yeast Res. 18 https://doi.org/10.1093/femsyr/foy063.

Müller, S., Harms, H., Bley, T., 2010. Origin and analysis of microbial population heterogeneity in bioprocesses. Curr. Opin. Biotechnol. 21, 100-113. https://doi.org/ 10.1016/j.copbio.2010.01.002.

Nielsen, J., Keasling, J.D., 2016. Engineering cellular metabolism. Cell 164, 1185-1197. https://doi.org/10.1016/j.cell.2016.02.004.
Nijkamp, J.F., van den Broek, M.A., Geertman, J.-M.A., Reinders, M.J.T., Daran, J.-M.G., de Ridder, D., 2012. De novo detection of copy number variation by co-assembly. Bioinformatics 28, 3195-3202. https://doi.org/10.1093/bioinformatics/bts601.

Redden, H., Alper, H.S., 2015. The development and characterization of synthetic minimal yeast promoters. Nat. Commun. 6, 7810. https://doi.org/10.1038/ ncomms8810.

Regenberg, B., Grotkjaer, T., Winther, O., Fausbøll, A., Akesson, M., Bro, C., Hansen, L.K., Brunak, S., Nielsen, J., 2006. Growth-rate regulated genes have profound impact on interpretation of transcriptome profiling in Saccharomyces cerevisiae. Genome Biol. 7, R107. https://doi.org/10.1186/gb-2006-7-11-r107.

Rugbjerg, P., Myling-Petersen, N., Porse, A., Sarup-Lytzen, K., Sommer, M.O.A., 2018a. Diverse genetic error modes constrain large-scale bio-based production. Nat. Commun. 9, 787. https://doi.org/10.1038/s41467-018-03232-w.

Rugbjerg, P., Sarup-Lytzen, K., Nagy, M., Sommer, M.O.A., 2018b. Synthetic addiction extends the productive life time of engineered Escherichia coli populations. Proc. Natl. Acad. Sci. U.S.A. 115, 2347-2352. https://doi.org/10.1073/pnas.1718622115.

Rugbjerg, P., Sommer, M.O.A., 2019. Overcoming genetic heterogeneity in industrial fermentations. Nat. Biotechnol. 37, 869-876. https://doi.org/10.1038/s41587-0190171-6.

Salazar, A.N., Gorter de Vries, A.R., van den Broek, M., Wijsman, M., de la Torre Cortés, P., Brickwedde, A., Brouwers, N., Daran, J.-M.G., Abeel, T., 2017. Nanopore sequencing enables near-complete de novo assembly of Saccharomyces cerevisiae reference strain CEN.PK113-7D. FEMS Yeast Res. 17 https://doi.org/10.1093/ femsyr/fox074.

Snoek, T., Romero-Suarez, D., Zhang, J., Ambri, F., Skjoedt, M.L., Sudarsan, S., Jensen, M.K., Keasling, J.D., 2018. An orthogonal and pH-tunable sensor-selector for muconic acid biosynthesis in yeast. ACS Synth. Biol. 7, 995-1003. https://doi.org/ 10.1021/acssynbio.7b00439.

Strucko, T., Buron, L.D., Jarczynska, Z.D., Nødvig, C.S., Mølgaard, L., Halkier, B.A., Mortensen, U.H., 2017. CASCADE, a platform for controlled gene amplification for high, tunable and selection-free gene expression in yeast. Sci. Rep. 7, 41431. https:// doi.org/10.1038/srep41431.

Strucko, T., Magdenoska, O., Mortensen, U.H., 2015. Benchmarking two commonly used Saccharomyces cerevisiae strains for heterologous vanillin- $\beta$-glucoside production. Metab. Eng. Commun. 2, 99-108. https://doi.org/10.1016/j.meteno.2015.09.001.

Tao, R., Zhao, Y., Chu, H., Wang, A., Zhu, J., Chen, X., Zou, Y., Shi, M., Liu, R., Su, N., Du, J., Zhou, H.-M., Zhu, L., Qian, X., Liu, H., Loscalzo, J., Yang, Y., 2017. Genetically encoded fluorescent sensors reveal dynamic regulation of NADPH metabolism. Nat. Methods 14, 720-728. https://doi.org/10.1038/nmeth.4306.

Verduyn, C., Postma, E., Scheffers, W.A., Van Dijken, J.P., 1992. Effect of benzoic acid on metabolic fluxes in yeasts: a continuous-culture study on the regulation of respiration and alcoholic fermentation. Yeast 8, 501-517. https://doi.org/10.1002/ yea.320080703.

Verhoeven, M.D., Lee, M., Kamoen, L., van den Broek, M., Janssen, D.B., Daran, J.-M.G., van Maris, A.J.A., Pronk, J.T., 2017. Mutations in PMR1 stimulate xylose isomerase activity and anaerobic growth on xylose of engineered Saccharomyces cerevisiae by influencing manganese homeostasis. Sci. Rep. 7, 46155. https://doi.org/10.1038/ srep46155.

Wang, T., Dunlop, M.J., 2019. Controlling and exploiting cell-to-cell variation in metabolic engineering. Curr. Opin. Biotechnol. 57, 10-16. https://doi.org/10.1016/ j.copbio.2018.08.013.

Weber, C., Brückner, C., Weinreb, S., Lehr, C., Essl, C., Boles, E., 2012. Biosynthesis of cis, cis-muconic acid and its aromatic precursors, catechol and protocatechuic acid, from renewable feedstocks by Saccharomyces cerevisiae. Appl. Environ. Microbiol. 78, 8421-8430. https://doi.org/10.1128/AEM.01983-12.

Wu, G., Yan, Q., Jones, J.A., Tang, Y.J., Fong, S.S., Koffas, M.A.G., 2016. Metabolic burden: cornerstones in synthetic biology and metabolic engineering applications. Trends Biotechnol. 34, 652-664. https://doi.org/10.1016/j.tibtech.2016.02.010.

Xiao, Y., Bowen, C.H., Liu, D., Zhang, F., 2016. Exploiting nongenetic cell-to-cell variation for enhanced biosynthesis. Nat. Chem. Biol. 12, 339-344. https://doi.org/ 10.1038/nchembio.2046.

Zhang, J., Sonnenschein, N., Pihl, T.P.B., Pedersen, K.R., Jensen, M.K., Keasling, J.D., 2016. Engineering an NADPH/NADP + redox biosensor in yeast. ACS Synth. Biol. 5, 1546-1556. https://doi.org/10.1021/acssynbio.6b00135. 